\title{
TELESCOPING METHOD, DERIVATIVE OPERATORS AND HARMONIC NUMBER IDENTITIES
}

\author{
${ }^{A}$ CHUANAN WEI, ${ }^{B}$ DIANXUAN GONG \\ ${ }^{A}$ Department of Information Technology \\ Hainan Medical College, Haikou 571101, China \\ ${ }^{B}$ College of Sciences \\ Hebei Polytechnic University, Tangshan 063009, China
}

\begin{abstract}
In terms of the telescoping method, a simple binomial sum is given. By applying the derivative operators to the equation just mentioned, we establish several general harmonic number identities including some known results.
\end{abstract}

\section{INTRODUCTION}

For $n \in \mathbb{N}_{0}$, define the harmonic numbers by

$$
H_{0}=0 \quad \text { and } \quad H_{n}=\sum_{k=1}^{n} \frac{1}{k} \quad \text { when } \quad n=1,2, \cdots .
$$

There exist many elegant identities involving harmonic numbers. They can be found in the papers [1]-[10].

For a function $f(x, y)$, define respectively the derivative operators $\mathcal{D}_{x}$ and $\mathcal{D}_{x y}^{2}$ by

$$
\begin{aligned}
& \mathcal{D}_{x} f(x, y)=\left.\frac{\partial}{\partial x} f(x, y)\right|_{x=0}, \\
& \mathcal{D}_{x y}^{2} f(x, y)=\left.\frac{\partial}{\partial y}\left\{\frac{\partial}{\partial x} f(x, y)\right\}\right|_{x=y=0} .
\end{aligned}
$$

Then it is not difficult to show the following two derivatives:

$$
\begin{aligned}
& \mathcal{D}_{x}\left(\begin{array}{c}
s+x \\
t
\end{array}\right)=\left(\begin{array}{l}
s \\
t
\end{array}\right)\left(H_{s}-H_{s-t}\right), \\
& \mathcal{D}_{x y}^{2} \frac{\left(\begin{array}{c}
s+x \\
t
\end{array}\right)}{\left(\begin{array}{c}
u+y \\
v
\end{array}\right)}=\frac{\left(\begin{array}{l}
s \\
t
\end{array}\right)}{\left(\begin{array}{l}
u \\
v
\end{array}\right)}\left(H_{s}-H_{s-t}\right)\left(H_{u-v}-H_{u}\right),
\end{aligned}
$$

where $s, t, u, v \in N_{0}$ with $t \leq s$ and $v \leq u$.

For a complex sequence $\left\{\tau_{k}\right\}_{k \in \mathbb{Z}}$, define the difference operator by

$$
\nabla \tau_{k}=\tau_{k}-\tau_{k-1} .
$$

Then we have the following relation:

$$
\nabla \frac{\left(\begin{array}{c}
x+k+1 \\
k
\end{array}\right)}{\left(\begin{array}{c}
y+k \\
k
\end{array}\right)}=\frac{\left(\begin{array}{c}
x+k \\
k
\end{array}\right)}{\left(\begin{array}{c}
y+k \\
k
\end{array}\right)} \frac{x-y+1}{x+1} .
$$

2010 Mathematics Subject Classification: Primary 05A19 and Secondary 40A25

Key words and phrases. Telescoping method; Derivative operator; Harmonic number identity.

Email addresses: weichuanan@yahoo.com.cn (C. Wei), gongdianxuan@yahoo.com.cn (D. Gong). 
Combining the last equation and the telescoping method:

$$
\sum_{k=1}^{n} \nabla \tau_{k}=\tau_{n}-\tau_{0}
$$

we obtain the simple binomial sum:

$$
\sum_{k=1}^{n} \frac{\left(\begin{array}{c}
x+k \\
k
\end{array}\right)}{\left(\begin{array}{c}
(+k \\
k
\end{array}\right)}=\frac{\left(\begin{array}{c}
x+n+1 \\
n
\end{array}\right)}{\left(\begin{array}{c}
y+n \\
n
\end{array}\right)} \frac{x+1}{x-y+1}-\frac{x+1}{x-y+1} .
$$

By applying the derivative operators to (1), several general harmonic number identities including some known results will be established in the next two sections.

\section{HARMONIC NUMBER IDENTITIES}

§2.1. Performing the replacement $y \rightarrow y+p$ for (11) with $p \in N_{0}$, we have

$$
\sum_{k=1}^{n} \frac{\left(\begin{array}{c}
x+k \\
k
\end{array}\right)}{\left(\begin{array}{c}
y+p+k \\
k
\end{array}\right)}=\frac{\left(\begin{array}{c}
x+n+1 \\
n
\end{array}\right)}{\left(\begin{array}{c}
y+p+n \\
n
\end{array}\right)} \frac{x+1}{x-y-p+1}-\frac{x+1}{x-y-p+1} .
$$

Applying the derivative operator $\mathcal{D}_{y}$ to the last equation, we establish the theorem.

Theorem 1. For $x \in \mathbb{C}$ and $p \in \mathbb{N}_{0}$, there holds the harmonic number identity:

$$
\sum_{k=1}^{n} \frac{\left(\begin{array}{c}
x+k \\
k
\end{array}\right)}{\left(\begin{array}{c}
p+k \\
k
\end{array}\right)} H_{p+k}=\frac{x+1}{x-p+1}\left\{\frac{\left(\begin{array}{c}
x+n+1 \\
n
\end{array}\right)}{\left(\begin{array}{c}
p+n \\
n
\end{array}\right)}\left(H_{p+n}-\frac{1}{x-p+1}\right)-H_{p}+\frac{1}{x-p+1}\right\} .
$$

Letting $x=p$ in Theorem 1, we achieve the following equation.

Corollary 2. For $p \in \mathbb{N}_{0}$, there holds the harmonic number identity:

$$
\sum_{k=1}^{n} H_{p+k}=(p+n+1) H_{p+n}-(p+1) H_{p}-n .
$$

When $p=0$, Corollary 2 reduces to the known result (cf. [2, Equation (2.1)]):

$$
\sum_{k=1}^{n} H_{k}=(n+1) H_{n}-n
$$

Setting $p=n$ and $p=2 n$ in Corollary 2 respectively, we attain the two identities:

$$
\begin{aligned}
& \sum_{k=1}^{n} H_{n+k}=(2 n+1) H_{2 n}-(n+1) H_{n}-n, \\
& \sum_{k=1}^{n} H_{2 n+k}=(3 n+1) H_{3 n}-(2 n+1) H_{2 n}-n .
\end{aligned}
$$

Making $x=p+1$ in Theorem 1 and considering the relation:

$$
\sum_{k=1}^{n} \frac{p+1+k}{p+1} H_{p+k}=\sum_{k=1}^{n}\left(1+\frac{k}{p+1}\right) H_{p+k},
$$

we get the following equation by using Corollary 2 ,

Corollary 3. For $p \in \mathbb{N}_{0}$, there holds the harmonic number identity:

$$
\sum_{k=1}^{n} k H_{p+k}=\frac{(n-p)(p+n+1)}{2} H_{p+n}+\frac{p(p+1)}{2} H_{p}-\frac{n(n-2 p-1)}{4} .
$$


When $p=0$, Corollary 3 reduces to the result due to Chen et al. [2, Equation (2.2)]:

$$
\sum_{k=1}^{n} k H_{k}=\frac{n(n+1)}{2} H_{n}-\frac{(n-1) n}{4} .
$$

Taking $p=n$ and $p=2 n$ in Corollary 3 respectively, we gain the two identities:

$$
\begin{aligned}
& \sum_{k=1}^{n} k H_{n+k}=\frac{n(n+1)}{2}\left(H_{n}+\frac{1}{2}\right), \\
& \sum_{k=1}^{n} k H_{2 n+k}=\frac{n(3 n+1)}{4}\left(1-2 H_{3 n}\right)+n(2 n+1) H_{2 n} .
\end{aligned}
$$

Letting $x=p+2$ in Theorem 1 and considering the relation:

$$
\sum_{k=1}^{n} \frac{(p+1+k)(p+2+k)}{(p+1)(p+2)} H_{p+k}=\sum_{k=1}^{n}\left\{1+\frac{(2 p+3) k}{(p+1)(p+2)}+\frac{k^{2}}{(p+1)(p+2)}\right\} H_{p+k},
$$

we achieve the following equation by using Corollaries 2 and 3 ,

Corollary 4. For $p \in \mathbb{N}_{0}$, there holds the harmonic number identity:

$$
\begin{aligned}
\sum_{k=1}^{n} k^{2} H_{p+k} & =\frac{(p+n+1)\left(2 n^{2}+n-2 p n+p+2 p^{2}\right)}{6} H_{p+n} \\
& -\frac{p(p+1)(2 p+1)}{6} H_{p}-\frac{n\left(4 n^{2}-3 n-6 p n+12 p+12 p^{2}-1\right)}{36} .
\end{aligned}
$$

When $p=0$, Corollary 4 reduces to the result due to Chen et al. [2, Equation (2.3)]:

$$
\sum_{k=1}^{n} k^{2} H_{k}=\frac{n(n+1)(2 n+1)}{6} H_{n}-\frac{(n-1) n(4 n+1)}{36} .
$$

When $p=n$, Corollary 4 reduces to the known result (cf. [2, Equation (2.7)]):

$$
\sum_{k=1}^{n} k^{2} H_{n+k}=\frac{n(n+1)(2 n+1)}{6}\left(2 H_{2 n}-H_{n}\right)-\frac{n(n+1)(10 n-1)}{36} .
$$

We remark that Chyzak [4] and Schneider [8] proved the last equation by an extension of Zeilberger's algorithm and Karr's algorithm respectively. Setting $p=2 n$ in Corollary 4, we attain the identity:

$$
\begin{aligned}
\sum_{k=1}^{n} k^{2} H_{2 n+k} & =\frac{n(2 n+1)(3 n+1)}{2} H_{3 n} \\
& -\frac{n(2 n+1)(4 n+1)}{3} H_{2 n}-\frac{n\left(40 n^{2}+21 n-1\right)}{36} .
\end{aligned}
$$

Choosing $x=-n-1$ in Theorem 1, we recover the result due to Sofo [9, Corollary 1]:

$$
\sum_{k=1}^{n}(-1)^{k}\left(\begin{array}{l}
n \\
k
\end{array}\right) \frac{H_{p+k}}{\left(\begin{array}{c}
p+k \\
k
\end{array}\right)}=\frac{-n}{p+n}\left(H_{p}+\frac{1}{p+n}\right) .
$$

Choosing $x=-n$ in Theorem 1 and considering the relation:

$$
\sum_{k=1}^{n}(-1)^{k}\left(\begin{array}{c}
n-1 \\
k
\end{array}\right) \frac{H_{p+k}}{\left(\begin{array}{c}
p+k \\
k
\end{array}\right)}=\sum_{k=1}^{n}(-1)^{k}\left(\begin{array}{l}
n \\
k
\end{array}\right)\left(1-\frac{k}{n}\right) \frac{H_{p+k}}{\left(\begin{array}{c}
p+k \\
k
\end{array}\right)},
$$

we recover, by using (2), the result due to Sofo [9, Corollary 3]:

$$
\sum_{k=1}^{n}(-1)^{k}\left(\begin{array}{l}
n \\
k
\end{array}\right) \frac{k H_{p+k}}{\left(\begin{array}{c}
p+k \\
k
\end{array}\right)}=\frac{n\left(n^{2}-n-p^{2}\right)}{(p+n)^{2}(p+n-1)^{2}}-\frac{p n H_{p}}{(p+n)(p+n-1)} .
$$


Choosing $x=1-n$ in Theorem 1 and considering the relation:

$$
\sum_{k=1}^{n}(-1)^{k}\left(\begin{array}{c}
n-2 \\
k
\end{array}\right) \frac{H_{p+k}}{\left(\begin{array}{c}
p+k \\
k
\end{array}\right)}=\sum_{k=1}^{n}(-1)^{k}\left(\begin{array}{l}
n \\
k
\end{array}\right)\left\{1-\frac{(2 n-1) k}{n(n-1)}+\frac{k^{2}}{n(n-1)}\right\} \frac{H_{p+k}}{\left(\begin{array}{c}
p+k \\
k
\end{array}\right)},
$$

we recover, by using (2) and (3), the result due to Chu[3, Corollary 1.8]:

$$
\begin{aligned}
\sum_{k=1}^{n}(-1)^{k}\left(\begin{array}{l}
n \\
k
\end{array}\right) \frac{k^{2} H_{p+k}}{\left(\begin{array}{c}
p+k \\
k
\end{array}\right)} & =\frac{p n(n-p) H_{p}}{(p+n)(p+n-1)(p+n-2)} \\
& -\frac{n^{3}}{(p+n)^{2}}+\frac{n(n-1)(2 n-1)}{(p+n-1)^{2}}-\frac{n(n-1)(n-2)}{(p+n-2)^{2}} .
\end{aligned}
$$

The case $p=0$ of (2), (3) and (4) read, respectively, as

$$
\begin{aligned}
& \sum_{k=1}^{n}(-1)^{k}\left(\begin{array}{l}
n \\
k
\end{array}\right) H_{k}=-\frac{1}{n} \quad \text { where } n>0, \\
& \sum_{k=1}^{n}(-1)^{k}\left(\begin{array}{l}
n \\
k
\end{array}\right) k H_{k}=\frac{1}{n-1} \quad \text { where } n>1, \\
& \sum_{k=1}^{n}(-1)^{k}\left(\begin{array}{l}
n \\
k
\end{array}\right) k^{2} H_{k}=-\frac{n}{(n-1)(n-2)} \quad \text { where } n>2 .
\end{aligned}
$$

The last three identities are very beautiful. Subsequently, we shall display several equations which include also (5), (6) and (7).

Making $x \rightarrow-x-n-1, p \rightarrow 0$ for Theorem 1, we get the following equation.

Corollary 5. For $x \in \mathbb{C}$, there holds the harmonic number identity:

$$
\sum_{k=1}^{n}(-1)^{k}\left(\begin{array}{c}
x+n \\
k
\end{array}\right) H_{k}=(-1)^{n}\left(\begin{array}{c}
x+n-1 \\
n
\end{array}\right)\left(H_{n}+\frac{1}{x+n}\right)-\frac{1}{x+n} .
$$

When $x=0$, Corollary 5 reduces to (5) exactly. Taking $x=n$ in Corollary 5 , we gain the identity:

$$
\sum_{k=1}^{n}(-1)^{k}\left(\begin{array}{c}
2 n \\
k
\end{array}\right) H_{k}=(-1)^{n}\left(\begin{array}{c}
2 n-1 \\
n
\end{array}\right)\left(H_{n}+\frac{1}{2 n}\right)-\frac{1}{2 n} \quad \text { where } n>0 .
$$

When $x=-\frac{1}{2}-n$, Corollary 5 reduces to the result due to Chen et al. 2, Example 2.3]:

$$
\sum_{k=1}^{n}\left(\begin{array}{c}
2 k \\
k
\end{array}\right) \frac{H_{k}}{4^{k}}=2-\frac{n+1}{4^{n}}\left(\begin{array}{c}
2 n+2 \\
n+1
\end{array}\right)+\frac{2 n+1}{4^{n}}\left(\begin{array}{c}
2 n \\
n
\end{array}\right) H_{n}
$$

Letting $x \rightarrow x-1$ for Corollary 5 and considering the relation:

$$
\sum_{k=1}^{n}(-1)^{k}\left(\begin{array}{c}
x-1+n \\
k
\end{array}\right) H_{k}=\sum_{k=1}^{n}(-1)^{k}\left(1-\frac{k}{x+n}\right)\left(\begin{array}{c}
x+n \\
k
\end{array}\right) H_{k},
$$

we achieve the following equation by using Corollary 5 ,

Corollary 6. For $x \in \mathbb{C}$, there holds the harmonic number identity:

$$
\begin{aligned}
\sum_{k=1}^{n}(-1)^{k}\left(\begin{array}{c}
x+n \\
k
\end{array}\right) k H_{k} & =\frac{(-1)^{n} n x}{x+n-1}\left(\begin{array}{c}
x+n \\
n
\end{array}\right) \\
& \times\left\{H_{n}+\frac{n^{2}+(n-1)(x-1)}{n(x+n)(x+n-1)}\right\}+\frac{1}{x+n-1} .
\end{aligned}
$$


When $x=0$, Corollary 6 reduces to (6) exactly. Setting $x=n$ and $x=-\frac{1}{2}-n$ in Corollary 6 respectively, we attain the two identities:

$$
\begin{aligned}
& \sum_{k=1}^{n}(-1)^{k}\left(\begin{array}{c}
2 n \\
k
\end{array}\right) k H_{k}=\frac{(-1)^{n} n^{2}}{2 n-1}\left(\begin{array}{c}
2 n \\
n
\end{array}\right)\left\{H_{n}+\frac{2 n^{2}-2 n+1}{2 n^{2}(2 n-1)}\right\}+\frac{1}{2 n-1}, \\
& \sum_{k=1}^{n}\left(\begin{array}{c}
2 k \\
k
\end{array}\right) \frac{k H_{k}}{4^{k}}=\frac{2 n+1}{9} \frac{\left(\begin{array}{c}
2 n \\
n
\end{array}\right)}{4^{n}}\left(3 n H_{n}-2 n+6\right)-\frac{2}{3} .
\end{aligned}
$$

Making $x \rightarrow x-1$ for Corollary 6 and considering the relation:

$$
\sum_{k=1}^{n}(-1)^{k}\left(\begin{array}{c}
x-1+n \\
k
\end{array}\right) k H_{k}=\sum_{k=1}^{n}(-1)^{k}\left(k-\frac{k^{2}}{x+n}\right)\left(\begin{array}{c}
x+n \\
k
\end{array}\right) H_{k},
$$

we get the following equation by using Corollary 6 .

Corollary 7. For $x \in \mathbb{C}$, there holds the harmonic number identity:

$$
\begin{aligned}
& \sum_{k=1}^{n}(-1)^{k}\left(\begin{array}{c}
x+n \\
k
\end{array}\right) k^{2} H_{k}=(-1)^{n} \frac{x\left(n^{2}-n+n x-1\right)}{(x+n-1)(x+n-2)}\left(\begin{array}{c}
x+n \\
n
\end{array}\right) \\
& \times\left\{n H_{n}+\frac{n(2 x+2 n-3)}{(x+n-1)(x+n-2)}-\frac{n^{2}+2 n-1}{n^{2}-n+n x-1}\right\}-\frac{x+n}{(x+n-1)(x+n-2)} .
\end{aligned}
$$

When $x=0$, Corollary 7 reduces to (7) exactly. Taking $x=n$ and $x=-\frac{1}{2}-n$ in Corollary 7 respectively, we gain the two identities:

$$
\begin{gathered}
\sum_{k=1}^{n}(-1)^{k}\left(\begin{array}{c}
2 n \\
k
\end{array}\right) k^{2} H_{k}=(-1)^{n} \frac{n^{2}(2 n+1)}{2(2 n-1)}\left(\begin{array}{c}
2 n \\
n
\end{array}\right)\left\{H_{n}+\frac{4 n^{3}-8 n^{2}+5 n-2}{2 n(n-1)\left(4 n^{2}-1\right)}\right\} \\
-\frac{n}{(n-1)(2 n-1)} \quad \text { where } n>1, \\
\sum_{k=1}^{n}\left(\begin{array}{c}
2 k \\
k
\end{array}\right) \frac{k^{2} H_{k}}{4^{k}}=\frac{(2 n+1)(3 n+2)}{15} \frac{\left(\begin{array}{c}
2 n \\
n
\end{array}\right)}{4^{n}}\left(n H_{n}+\frac{8 n-6}{9 n+6}-\frac{2 n}{5}\right)+\frac{2}{15} .
\end{gathered}
$$

§2.2. Employing the substitutions $x \rightarrow y+p, y \rightarrow x$ for (11) with $p \in N_{0}$, we have

$$
\sum_{k=1}^{n} \frac{\left(\begin{array}{c}
y+p+k \\
k
\end{array}\right)}{\left(\begin{array}{c}
x+k \\
k
\end{array}\right)}=\frac{\left(\begin{array}{c}
y+p+n+1 \\
n
\end{array}\right)}{\left(\begin{array}{c}
x+n \\
n
\end{array}\right)} \frac{y+p+1}{y-x+p+1}-\frac{y+p+1}{y-x+p+1} .
$$

Applying the derivative operator $\mathcal{D}_{y}$ to the last equation, we found the theorem.

Theorem 8. For $y \in \mathbb{C}$ and $p \in \mathbb{N}_{0}$, there holds the harmonic number identity:

$$
\begin{aligned}
\sum_{k=1}^{n} \frac{\left(\begin{array}{c}
p+k \\
k
\end{array}\right)}{\left(\begin{array}{c}
x+k \\
k
\end{array}\right)} H_{p+k} & =\frac{p+1}{p-x+1} \frac{\left(\begin{array}{c}
p+n+1 \\
n
\end{array}\right)}{\left(\begin{array}{c}
x+n \\
n
\end{array}\right)}\left(H_{p+n+1}-\frac{1}{p-x+1}\right) \\
& -\frac{p+1}{p-x+1} H_{p}+\frac{x}{(p-x+1)^{2}} .
\end{aligned}
$$

Of course, Corollaries 24 can also be implied by the theorem. Now, we shall derive other several results, which correspond to Corollaries 5.7, from Theorem 8 .

Letting $x \rightarrow-x-n-1, p \rightarrow 0$ for Theorem 8 , we achieve the following equation.

Corollary 9. For $x \in \mathbb{C}$, there holds the harmonic number identity:

$$
\sum_{k=1}^{n} \frac{(-1)^{k}}{\left(\begin{array}{c}
x+n \\
k
\end{array}\right)} H_{k}=\frac{n+1}{x+n+2} \frac{(-1)^{n}}{\left(\begin{array}{c}
x+n \\
n
\end{array}\right)}\left(H_{n+1}-\frac{1}{x+n+2}\right)-\frac{x+n+1}{(x+n+2)^{2}} .
$$


When $x=0$, Corollary 9 reduces to the interesting identity:

$$
\sum_{k=1}^{n} \frac{(-1)^{k}}{\left(\begin{array}{l}
n \\
k
\end{array}\right)} H_{k}=(-1)^{n} \frac{n+1}{n+2}\left(H_{n+1}-\frac{1}{n+2}\right)-\frac{n+1}{(n+2)^{2}} .
$$

Setting $x=n$ and $x=-\frac{1}{2}-n$ in Corollary 9 respectively, we attain the two identities:

$$
\begin{aligned}
& \sum_{k=1}^{n} \frac{(-1)^{k}}{\left(\begin{array}{c}
2 n \\
k
\end{array}\right)} H_{k}=\frac{(-1)^{n}}{4\left(\begin{array}{c}
2 n \\
n
\end{array}\right)}\left(2 H_{n+1}-\frac{1}{n+1}\right)-\frac{2 n+1}{4(n+1)^{2}}, \\
& \sum_{k=1}^{n} \frac{4^{k}}{\left(\begin{array}{c}
2 k \\
k
\end{array}\right)} H_{k}=\frac{2(n+1)}{3} \frac{4^{n}}{\left(\begin{array}{c}
2 n \\
n
\end{array}\right)}\left(H_{n+1}-\frac{2}{3}\right)-\frac{2}{9} .
\end{aligned}
$$

Making $x \rightarrow x+1$ for Corollary 9 and considering the relation:

$$
\sum_{k=1}^{n} \frac{(-1)^{k}}{\left(\begin{array}{c}
x+n+1 \\
k
\end{array}\right)} H_{k}=\sum_{k=1}^{n} \frac{(-1)^{k}}{\left(\begin{array}{c}
x+n \\
k
\end{array}\right)}\left(1-\frac{k}{x+n+1}\right) H_{k},
$$

we get the following equation by using Corollary 9 ,

Corollary 10. For $x \in \mathbb{C}$, there holds the harmonic number identity:

$$
\begin{aligned}
\sum_{k=1}^{n} \frac{(-1)^{k}}{\left(\begin{array}{c}
x+n \\
k
\end{array}\right)} k H_{k} & =\left\{\frac{1+x+n(x+n+3)}{(x+n+2)(x+n+3)} H_{n+1}-\frac{x+n+1}{(x+n+2)^{2}}+\frac{x+1}{(x+n+3)^{2}}\right\} \\
& \times \frac{(-1)^{n}}{\left(\begin{array}{c}
x+n \\
n
\end{array}\right)}(n+1)-\left\{\frac{x+n+1}{(x+n+2)^{2}}-\frac{x+n+2}{(x+n+3)^{2}}\right\}(x+n+1) .
\end{aligned}
$$

When $x=0$, Corollary 10 reduces to the interesting identity:

$$
\begin{aligned}
\sum_{k=1}^{n} \frac{(-1)^{k}}{\left(\begin{array}{l}
n \\
k
\end{array}\right)} k H_{k} & =\left\{H_{n+1}+\frac{n}{n^{2}+3 n+1}-\frac{2 n+5+(-1)^{n}}{(n+2)(n+3)}\right\} \\
& \times \frac{(n+1)\left(n^{2}+3 n+1\right)(-1)^{n}}{(n+2)(n+3)} .
\end{aligned}
$$

Taking $x=n$ and $x=-\frac{1}{2}-n$ in Corollary 10 respectively, we gain the two identities:

$$
\begin{aligned}
\sum_{k=1}^{n} \frac{(-1)^{k}}{\left(\begin{array}{c}
2 n \\
k
\end{array}\right)} k H_{k}= & \left\{H_{n+1}+\frac{n}{2 n^{2}+4 n+1}-\frac{4 n+5}{(2 n+2)(2 n+3)}\right\} \\
& \times \frac{(-1)^{n}}{\left(\begin{array}{c}
2 n \\
n
\end{array}\right)} \frac{2 n^{2}+4 n+1}{4 n+6}-\frac{(2 n+1)\left(4 n^{2}+6 n+1\right)}{(2 n+2)^{2}(2 n+3)^{2}}, \\
\sum_{k=1}^{n} \frac{4^{k}}{\left(\begin{array}{c}
2 k \\
k
\end{array}\right)} k H_{k}= & \frac{2(n+1)(3 n+1)}{15} \frac{4^{n}}{\left(\begin{array}{c}
2 n \\
n
\end{array}\right)}\left(H_{n+1}-\frac{2}{9 n+3}-\frac{2}{5}\right)+\frac{2}{225} .
\end{aligned}
$$

Letting $x \rightarrow x+1$ for Corollary 10 and considering the relation:

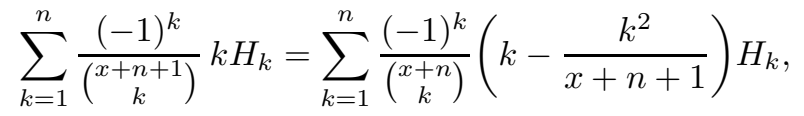

we achieve the following equation by using Corollary 10 .

Corollary 11. For $y \in \mathbb{C}$, there holds the harmonic number identity:

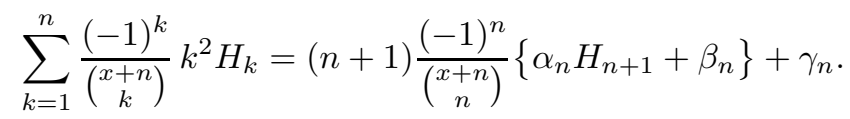


where

$$
\begin{aligned}
& \alpha_{n}=\frac{1}{x+n+2}-\frac{3 n+6}{x+n+3}+\frac{n^{2}+5 n+6}{x+n+4}, \\
& \beta_{n}=\frac{2 x+2 n+3}{(x+n+2)^{2}}-\frac{7 x+15+2 n(x+n+5)}{(x+n+3)^{2}}+\frac{5 x+14+n(2 x+n+8)}{(x+n+4)^{2}}, \\
& \gamma_{n}=\left\{\frac{2 x+2 n+3}{(x+n+2)^{2}}-\frac{5 x+5 n+12}{(x+n+3)^{2}}+\frac{3 x+3 n+10}{(x+n+4)^{2}}\right\}(x+n+1) .
\end{aligned}
$$

When $x=0$, Corollary 10 reduces to the interesting identity:

$$
\begin{aligned}
\sum_{k=1}^{n} \frac{(-1)^{k}}{\left(\begin{array}{l}
n \\
k
\end{array}\right)} k^{2} H_{k} & =\left\{\frac{n\left(n^{3}+7 n^{2}+14 n+7\right)}{(n+2)(n+3)(n+4)} H_{n+1}-\frac{n^{6}+14 n^{5}+77 n^{4}+208 n^{3}+279 n^{2}+160 n+24}{(n+2)^{2}(n+3)^{2}(n+4)^{2}}\right\} \\
& \times(n+1)(-1)^{n}-\frac{(n+1)\left(n^{4}+5 n^{3}-n^{2}-28 n-24\right)}{(n+2)^{2}(n+3)^{2}(n+4)^{2}} .
\end{aligned}
$$

Setting $x=n$ and $x=-\frac{1}{2}-n$ in Corollary 10 respectively, we attain the two identities:

$$
\begin{aligned}
\sum_{k=1}^{n} \frac{(-1)^{k}}{\left(\begin{array}{c}
2 n \\
k
\end{array}\right)} k^{2} H_{k} & =\left\{\frac{n(n+2)(2 n+1)}{2(n+1)(2 n+3)} H_{n+1}-\frac{4 n^{5}+28 n^{4}+69 n^{3}+71 n^{2}+28 n+3}{4(n+1)^{2}(n+2)(2 n+3)^{2}}\right\} \\
& \times \frac{(-1)^{n}}{\left(\begin{array}{c}
2 n \\
n
\end{array}\right)}(n+1)-\frac{n(n+2)\left(8 n^{3}+8 n^{2}-8 n-13\right)-6}{4(n+1)^{2}(n+2)^{2}(2 n+3)^{2}}, \\
\sum_{k=1}^{n} \frac{4^{k}}{\left(\begin{array}{c}
2 k \\
k
\end{array}\right)} k^{2} H_{k}= & \left(\frac{15 n^{2}+12 n-1}{210} H_{n+1}-\frac{225 n^{2}+432 n+34}{11025}\right) \frac{4^{n+1}}{\left(\begin{array}{c}
2 n \\
n
\end{array}\right)}(n+1)+\frac{346}{11025} .
\end{aligned}
$$

\section{Further HARMONiC NUMBER IDENTIties}

§3.1. Performing the replacements $x \rightarrow x+p, y \rightarrow y+q$ for (11) with $p, q \in N_{0}$, we have

$$
\sum_{k=1}^{n} \frac{\left(\begin{array}{c}
x+p+k \\
k
\end{array}\right)}{\left(\begin{array}{c}
y+q+k \\
k
\end{array}\right)}=\frac{\left(\begin{array}{c}
x+p+n+1 \\
n
\end{array}\right)}{\left(\begin{array}{c}
y+q+n \\
n
\end{array}\right)} \frac{x+p+1}{x-y+p-q+1}-\frac{x+p+1}{x-y+p-q+1} .
$$

Applying the derivative operator $\mathcal{D}_{x y}^{2}$ to the last equation and using Theorems 1 and 8 , we establish the theorem.

Theorem 12. For $p, q \in \mathbb{N}_{0}$, there holds the harmonic number identity:

$$
\begin{aligned}
\sum_{k=1}^{n} \frac{(p+k) !}{(q+k) !} H_{p+k} H_{q+k} & =\left\{(p-q+1) H_{p+n+1} H_{q+n}-H_{p+n+1}-H_{q+n}+\frac{2}{p-q+1}\right\} \\
& \times \frac{(p+n+1) !}{(q+n) !(p-q+1)^{2}}-\frac{(p+1) !}{q !(p-q+1)^{2}} \\
& \times\left\{(p-q+1) H_{p+1} H_{q}-H_{p+1}-H_{q}+\frac{2}{p-q+1}\right\} .
\end{aligned}
$$

Making $q=p$ in Theorem 12 , we get the following equation.

Corollary 13. For $p \in \mathbb{N}_{0}$, there holds the harmonic number identity:

$$
\sum_{k=1}^{n} H_{p+k}^{2}=(p+n+1) H_{p+n}^{2}-(2 p+2 n+1) H_{p+n}-(p+1) H_{p}^{2}+(2 p+1) H_{p}+2 n .
$$

When $p=0$, Corollary 13 reduces to the known result (cf. [2, Equation (2.8)]):

$$
\sum_{k=1}^{n} H_{k}^{2}=(n+1) H_{n}^{2}-(2 n+1) H_{n}+2 n
$$


Taking $p=n$ and $p=2 n$ in Corollary 13 respectively, we gain the two identities:

$$
\begin{aligned}
& \sum_{k=1}^{n} H_{n+k}^{2}=(2 n+1) H_{2 n}^{2}-(4 n+1) H_{2 n}-(n+1) H_{n}^{2}+(2 n+1) H_{n}+2 n, \\
& \sum_{k=1}^{n} H_{2 n+k}^{2}=(3 n+1) H_{3 n}^{2}-(6 n+1) H_{3 n}-(2 n+1) H_{2 n}^{2}+(4 n+1) H_{2 n}+2 n .
\end{aligned}
$$

Letting $q=p-1$ in Theorem 12 and considering the relation:

$$
\sum_{k=1}^{n}(p+k) H_{p+k} H_{p+k-1}=\sum_{k=1}^{n}(p+k) H_{p+k}^{2}-\sum_{k=1}^{n} H_{p+k},
$$

we achieve the following equation by using Corollaries 2 and 13 .

Corollary 14. For $p \in \mathbb{N}_{0}$, there holds the harmonic number identity:

$$
\begin{aligned}
\sum_{k=1}^{n} k H_{p+k}^{2} & =\frac{(n-p)(n+p+1)}{2} H_{p+n}^{2}-\frac{n^{2}-n-1-p(2 n+3 p+3)}{2} H_{p+n} \\
& +\frac{p(p+1)}{2} H_{p}^{2}-\frac{3 p^{2}+3 p+1}{2} H_{p}+\frac{n(n-6 p-3)}{4} .
\end{aligned}
$$

When $p=0$, Corollary 14 reduces to the interesting identity:

$$
\sum_{k=1}^{n} k H_{k}^{2}=\frac{n(n+1)}{2} H_{n}^{2}-\frac{n^{2}-n-1}{2} H_{n}+\frac{n(n-3)}{4} .
$$

Setting $p=n$ and $p=2 n$ in Corollary 14 respectively, we attain the two identities:

$$
\begin{aligned}
\sum_{k=1}^{n} k H_{n+k}^{2}= & \frac{(2 n+1)^{2}}{2} H_{2 n}+\frac{n(n+1)}{2} H_{n}^{2}-\frac{3 n^{2}+3 n+1}{2} H_{n}-\frac{n(5 n+3)}{4} \\
\sum_{k=1}^{n} k H_{2 n+k}^{2}= & -\frac{n(3 n+1)}{2} H_{3 n}^{2}+\frac{15 n^{2}+7 n+1}{2} H_{3 n}+n(2 n+1) H_{2 n}^{2} \\
& -\frac{12 n^{2}+6 n+1}{2} H_{2 n}-\frac{n(11 n+3)}{4}
\end{aligned}
$$

Making $q=p-2$ in Theorem 12 and considering the relation:

$$
\begin{aligned}
\sum_{k=1}^{n}(p+k)(p+k-1) H_{p+k} H_{p+k-2} & =\sum_{k=1}^{n}\left\{p(p-1)+(2 p-1) k+k^{2}\right\} H_{p+k}^{2} \\
& -\sum_{k=1}^{n}\{(2 p-1)+2 k\} H_{p+k},
\end{aligned}
$$

we get the following equation by using Corollaries 2, 3, 13 and 14.

Corollary 15. For $p \in \mathbb{N}_{0}$, there holds the harmonic number identity:

$$
\begin{aligned}
\sum_{k=1}^{n} k^{2} H_{p+k}^{2} & =\frac{(p+n+1)\left(2 n^{2}+n-2 p n+p+2 p^{2}\right)}{6} H_{p+n}^{2}-\frac{p(p+1)(2 p+1)}{6} H_{p}^{2} \\
& -\frac{4 n^{3}-3 n^{2}-6 p n^{2}-n+12 p n+12 p^{2} n+3+17 p+33 p^{2}+22 p^{3}}{18} H_{p+n} \\
& +\frac{(2 p+1)\left(11 p^{2}+11 p+3\right)}{18} H_{p}+\frac{n\left(8 n^{2}-15 n-30 p n+25+132 p+132 p^{2}\right)}{108} .
\end{aligned}
$$

When $p=0$, Corollary 15 reduces to the interesting identity:

$$
\sum_{k=1}^{n} k^{2} H_{k}^{2}=\frac{n(n+1)(2 n+1)}{6} H_{n}^{2}-\frac{4 n^{3}-3 n^{2}-n+3}{18} H_{n}+\frac{n\left(8 n^{2}-15 n+25\right)}{108} .
$$


Taking $p=n$ and $p=2 n$ in Corollary 15 respectively, we gain the two identities:

$$
\begin{aligned}
\sum_{k=1}^{n} k^{2} H_{n+k}^{2}= & \frac{n(n+1)(2 n+1)}{3} H_{2 n}^{2}-\frac{32 n^{3}+42 n^{2}+16 n+3}{18} H_{2 n}-\frac{n(n+1)(2 n+1)}{6} H_{n}^{2} \\
& +\frac{(2 n+1)\left(11 n^{2}+11 n+3\right)}{18} H_{n}+\frac{n\left(110 n^{2}+117 n+25\right)}{108} \\
\sum_{k=1}^{n} k^{2} H_{2 n+k}^{2} & =\frac{n(2 n+1)(3 n+1)}{2} H_{3 n}^{2}-\frac{72 n^{3}+51 n^{2}+11 n+1}{6} H_{3 n}-\frac{n(2 n+1)(4 n+1)}{3} H_{2 n}^{2} \\
& +\frac{(4 n+1)\left(44 n^{2}+22 n+3\right)}{18} H_{2 n}+\frac{n\left(476 n^{2}+249 n+25\right)}{108} .
\end{aligned}
$$

§3.2. Employing the substitutions $x \rightarrow-x-p-n-1, y \rightarrow y+q$ for (11) with $p, q \in N_{0}$, we have

$$
\sum_{k=1}^{n}(-1)^{k} \frac{\left(\begin{array}{c}
x+p+n \\
k
\end{array}\right)}{\left(\begin{array}{c}
y+q+k \\
k
\end{array}\right)}=(-1)^{n} \frac{\left(\begin{array}{c}
x+p+n-1 \\
n
\end{array}\right)}{\left(\begin{array}{c}
y+q+n \\
n
\end{array}\right)} \frac{x+p+n}{x+y+p+q+n}-\frac{x+p+n}{x+y+p+q+n} .
$$

Applying the derivative operator $\mathcal{D}_{x y}^{2}$ to the last equation and using Theorem 1 , we found the theorem.

Theorem 16. For $p, q \in \mathbb{N}_{0}$, there holds the harmonic number identity:

$$
\begin{aligned}
& \sum_{k=1}^{n}(-1)^{k}\left(\begin{array}{c}
p+q+n \\
q+k
\end{array}\right) H_{p+n-k} H_{q+k} \\
& =\left\{H_{p} H_{q+n}+\frac{H_{p}}{p+q+n}-\frac{(q+n) H_{q+n}}{p(p+q+n)}+\frac{p-q-n}{p(p+q+n)^{2}}\right\} \\
& \times\left(\begin{array}{c}
p+q+n \\
p
\end{array}\right) \frac{(-1)^{n} p}{p+q+n}-\left(\begin{array}{c}
p+q+n \\
q
\end{array}\right) \frac{p+n}{p+q+n} \\
& \times\left\{H_{p+n} H_{q}+\frac{H_{p+n}}{p+q+n}-\frac{q H_{q}}{(p+n)(p+q+n)}+\frac{p-q+n}{(p+n)(p+q+n)^{2}}\right\} .
\end{aligned}
$$

Letting $q=0$ in Theorem 16, we achieve the following equation.

Corollary 17. For $p \in \mathbb{N}_{0}$, there holds the harmonic number identity:

$$
\begin{aligned}
& \sum_{k=1}^{n}(-1)^{k}\left(\begin{array}{c}
p+n \\
k
\end{array}\right) H_{k} H_{p+n-k}=(-1)^{n}\left(\begin{array}{c}
p+n-1 \\
n
\end{array}\right) \\
& \times\left\{H_{p} H_{n}+\frac{H_{p}}{p+n}-\frac{n H_{n}}{p(p+n)}+\frac{p-n}{p(p+n)^{2}}\right\}-\frac{1}{p+n}\left\{H_{p+n}+\frac{1}{p+n}\right\} .
\end{aligned}
$$

When $p=0$, Corollary 17 reduces to the interesting identity:

$$
\sum_{k=1}^{n}(-1)^{k}\left(\begin{array}{l}
n \\
k
\end{array}\right) H_{k} H_{n-k}=\frac{(-1)^{n+1}-1}{n}\left(H_{n}+\frac{1}{n}\right) \text { where } n>0 .
$$

Setting $p=n$ in Corollary 17, we attain the identity:

$$
\sum_{k=1}^{n}(-1)^{k}\left(\begin{array}{c}
2 n \\
k
\end{array}\right) H_{k} H_{2 n-k}=(-1)^{n}\left(\begin{array}{c}
2 n-1 \\
n
\end{array}\right) H_{n}^{2}-\frac{1}{2 n}\left(H_{2 n}+\frac{1}{2 n}\right) \quad \text { where } n>0 .
$$

Making $p \rightarrow p-1$ for Corollary 17 and considering the relation:

$$
\begin{aligned}
\sum_{k=1}^{n}(-1)^{k}\left(\begin{array}{c}
p+n-1 \\
k
\end{array}\right) H_{k} H_{p+n-1-k} & =\sum_{k=1}^{n}(-1)^{k}\left(\begin{array}{c}
p+n \\
k
\end{array}\right)\left(1-\frac{k}{p+n}\right) H_{k} H_{p+n-k} \\
& -\frac{1}{p+n} \sum_{k=1}^{n}(-1)^{k}\left(\begin{array}{c}
p+n \\
k
\end{array}\right) H_{k},
\end{aligned}
$$


we get the following equation by using Corollaries 5 and 17 .

Corollary 18. For $p \in \mathbb{N}_{0}$, there holds the harmonic number identity:

$$
\begin{aligned}
& \sum_{k=1}^{n}(-1)^{k}\left(\begin{array}{c}
p+n \\
k
\end{array}\right) k H_{k} H_{p+n-k}=\frac{1}{p+n-1}\left\{H_{p+n}+\frac{1}{p+n-1}\right\}+\frac{(-1)^{n} p n}{p+n-1}\left(\begin{array}{c}
p+n \\
n
\end{array}\right) \\
& \times\left\{H_{p} H_{n}+\frac{n^{2}+(p-1)(n-1)}{n(p+n)(p+n-1)} H_{p}-\frac{n-1}{p(p+n-1)} H_{n}-\frac{p+n-1}{p(p+n)^{2}}-\frac{n-2 p n+p-1}{p n(p+n-1)^{2}}\right\} .
\end{aligned}
$$

When $p=0$, Corollary 18 reduces to the interesting identity:

$$
\begin{aligned}
\sum_{k=1}^{n}(-1)^{k}\left(\begin{array}{l}
n \\
k
\end{array}\right) k H_{k} H_{n-k} & =\frac{1}{n-1}\left(H_{n}+\frac{1}{n-1}\right) \\
& +(-1)^{n} \frac{n}{1-n}\left\{H_{n}+\frac{n^{2}-n+1}{n^{2}(n-1)}\right\} \quad \text { where } n>1 .
\end{aligned}
$$

Taking $p=n$ in Corollary 18, we gain the identity:

$$
\begin{aligned}
\sum_{k=1}^{n}(-1)^{k}\left(\begin{array}{c}
2 n \\
k
\end{array}\right) k H_{k} H_{2 n-k} & =\frac{1}{2 n-1}\left(H_{2 n}+\frac{1}{2 n-1}\right)+(-1)^{n} \frac{n^{2}}{2 n-1}\left(\begin{array}{c}
2 n \\
n
\end{array}\right) \\
& \times\left\{H_{n}^{2}+\frac{H_{n}}{2 n^{2}(2 n-1)}+\frac{4 n^{2}-2 n+1}{4 n^{3}(2 n-1)^{2}}\right\} \quad \text { where } n>0 .
\end{aligned}
$$

Letting $p \rightarrow p-1$ for Corollary 18 and considering the relation:

$$
\begin{aligned}
\sum_{k=1}^{n}(-1)^{k}\left(\begin{array}{c}
p+n-1 \\
k
\end{array}\right) k H_{k} H_{p+n-1-k} & =\sum_{k=1}^{n}(-1)^{k}\left(\begin{array}{c}
p+n \\
k
\end{array}\right)\left(k-\frac{k^{2}}{p+n}\right) H_{k} H_{p+n-k} \\
& -\frac{1}{p+n} \sum_{k=1}^{n}(-1)^{k}\left(\begin{array}{c}
p+n \\
k
\end{array}\right) k H_{k},
\end{aligned}
$$

we achieve the following equation by using Corollaries 6 and 18 .

Corollary 19. For $p \in \mathbb{N}_{0}$, there holds the harmonic number identity:

$$
\begin{aligned}
& \sum_{k=1}^{n}(-1)^{k}\left(\begin{array}{c}
p+n \\
k
\end{array}\right) k^{2} H_{k} H_{p+n-k}=(-1)^{n}\left(\begin{array}{c}
p+n \\
n
\end{array}\right)\left\{\theta_{n} H_{n} H_{p}-\lambda_{n} H_{n}-\mu_{n} H_{p}-\nu_{n}\right\} \\
& -\varepsilon_{n} H_{p+n}-\eta_{n} \\
& \mu_{n}=\frac{p\left(n^{2}+2 n-1\right)}{(p+n-1)(p+n-2)}-\frac{p n(2 p+2 n-3)\left(n^{2}+p n-n-1\right)}{(p+n-1)^{2}(p+n-2)^{2}} \text {, } \\
& \nu_{n}=\frac{2 n^{2}-4 n+1}{(p+n-1)^{2}}+\frac{2 n(n-1)}{(p+n-1)^{3}}-\frac{3 n^{2}-6 n+2}{(p+n-2)^{2}}+\frac{2 n(n-1)(n-2)}{(p+n-2)^{3}}, \\
& \varepsilon_{n}=\frac{p+n}{(p+n-1)(p+n-2)}, \quad \eta_{n}=\frac{(p+n)^{2}-2}{(p+n-1)^{2}(p+n-2)^{2}} .
\end{aligned}
$$

When $p=0$, Corollary 19 reduces to the interesting identity:

$$
\begin{aligned}
\sum_{k=1}^{n}(-1)^{k}\left(\begin{array}{c}
n \\
k
\end{array}\right) k^{2} H_{k} H_{n-k} & =(-1)^{n}\left\{\frac{n+n^{2}-n^{3}}{(n-1)(n-2)} H_{n}-\frac{n^{4}-4 n^{3}+6 n^{2}-4 n+2}{(n-1)^{2}(n-2)^{2}}\right\} \\
& -\frac{n}{(n-1)(n-2)}\left\{H_{n}+\frac{n^{2}-2}{n(n-1)(n-2)}\right\} \quad \text { where } n>2 .
\end{aligned}
$$


Setting $p=n$ in Corollary 19, we attain the identity:

$$
\begin{aligned}
\sum_{k=1}^{n}(-1)^{k}\left(\begin{array}{c}
2 n \\
k
\end{array}\right) k^{2} H_{k} H_{2 n-k} & =(-1)^{n}\left(\begin{array}{c}
2 n \\
n
\end{array}\right)\left\{\frac{2 n^{3}+n^{2}}{4 n-2} H_{n}^{2}+\frac{n}{(2 n-1)^{2}} H_{n}+\frac{4 n^{2}-2 n+1}{2(2 n-1)^{3}}\right\} \\
& -\frac{n}{(n-1)(2 n-1)}\left\{H_{2 n}+\frac{2 n^{2}-1}{n(2 n-1)(2 n-2)}\right\} \quad \text { where } n>1 .
\end{aligned}
$$

§3.3. Performing the replacements $x \rightarrow x+q, y \rightarrow-y-p-n-1$ for (1) with $p, q \in N_{0}$, we have

$$
\sum_{k=1}^{n}(-1)^{k} \frac{\left(\begin{array}{c}
x+q+k \\
k
\end{array}\right)}{\left(\begin{array}{c}
y+p+n \\
k
\end{array}\right)}=(-1)^{n} \frac{\left(\begin{array}{c}
x+q+n+1 \\
n
\end{array}\right)}{\left(\begin{array}{c}
y+p+n \\
n
\end{array}\right)} \frac{x+q+1}{x+y+p+q+n+2}-\frac{x+q+1}{x+y+p+q+n+2} .
$$

Applying the derivative operator $\mathcal{D}_{x y}^{2}$ to the last equation and using Theorem 8 , we establish the theorem.

Theorem 20. For $p, q \in \mathbb{N}_{0}$, there holds the harmonic number identity:

$$
\begin{aligned}
& \sum_{k=1}^{n} \frac{(-1)^{k}}{\left(\begin{array}{c}
p+q+n \\
q+k
\end{array}\right)} H_{p+n-k} H_{q+k} \\
& =\left\{H_{p} H_{q+n+1}-\frac{H_{p}}{p+q+n+2}-\frac{H_{q+n+1}}{p+q+n+2}+\frac{2}{(p+q+n+2)^{2}}\right\} \\
& \times \frac{(-1)^{n}}{\left(\begin{array}{c}
p+q+n \\
p
\end{array}\right)} \frac{q+n+1}{p+q+n+2}-\frac{1}{\left(\begin{array}{c}
p+q+n \\
q
\end{array}\right)} \frac{q+1}{p+q+n+2} \\
& \times\left\{H_{p+n} H_{q+1}-\frac{H_{p+n}}{p+q+n+2}-\frac{H_{q+1}}{p+q+n+2}+\frac{2}{(p+q+n+2)^{2}}\right\} .
\end{aligned}
$$

Making $q=0$ in Theorem 20 , we get the equation.

Corollary 21. For $p \in \mathbb{N}_{0}$, there holds the harmonic number identity:

$$
\begin{aligned}
& \sum_{k=1}^{n} \frac{(-1)^{k}}{\left(\begin{array}{c}
p+n \\
k
\end{array}\right)} H_{k} H_{p+n-k}=\left\{H_{p} H_{n+1}-\frac{H_{p}}{p+n+2}-\frac{H_{n+1}}{p+n+2}+\frac{2}{(p+n+2)^{2}}\right\} \\
& \times \frac{(-1)^{n}}{\left(\begin{array}{c}
p+n \\
p
\end{array}\right)} \frac{n+1}{p+n+2}-\frac{p+n+1}{(p+n+2)^{2}}\left\{H_{p+n}-\frac{p+n}{(p+n+1)(p+n+2)}\right\} .
\end{aligned}
$$

When $p=0$, Corollary 21 reduces to the interesting identity:

$$
\sum_{k=1}^{n} \frac{(-1)^{k}}{\left(\begin{array}{l}
n \\
k
\end{array}\right)} H_{k} H_{n-k}=\frac{n+1}{(n+2)^{2}}\left\{\frac{2}{n+2}-H_{n+1}\right\}\left\{1+(-1)^{n}\right\} .
$$

Taking $p=n$ in Corollary 21, we gain the identity:

$$
\sum_{k=1}^{n} \frac{(-1)^{k}}{\left(\begin{array}{c}
2 n \\
k
\end{array}\right)} H_{k} H_{2 n-k}=\frac{(-1)^{n}}{\left(\begin{array}{c}
2 n \\
n
\end{array}\right)} \frac{H_{n}^{2}}{2}-\frac{2 n+1}{(2 n+2)^{2}}\left\{H_{2 n}-\frac{n}{(n+1)(2 n+1)}\right\} .
$$

Letting $p \rightarrow p+1$ for Corollary 21 and considering the relation:

$$
\begin{aligned}
\sum_{k=1}^{n} \frac{(-1)^{k}}{\left(\begin{array}{c}
p+n+1 \\
k
\end{array}\right)} H_{k} H_{p+n+1-k} & =\sum_{k=1}^{n} \frac{(-1)^{k}}{\left(\begin{array}{c}
p+n \\
k
\end{array}\right)}\left(1-\frac{k}{p+n+1}\right) H_{k} H_{p+n-k} \\
& +\frac{1}{p+n+1} \sum_{k=1}^{n} \frac{(-1)^{k}}{\left(\begin{array}{c}
p+n \\
k
\end{array}\right)} H_{k},
\end{aligned}
$$

we achieve the following equation by using Corollaries 9 and 21$]$ 
Corollary 22. For $p \in N_{0}$, there holds the harmonic number identity:

$$
\begin{aligned}
\sum_{k=1}^{n} \frac{(-1)^{k}}{\left(\begin{array}{c}
p+n \\
k
\end{array}\right)} k H_{k} H_{p+n-k} & =(-1)^{n} \frac{n+1}{\left(\begin{array}{c}
p+n \\
n
\end{array}\right)}\left\{A_{n} H_{n+1} H_{p}+B_{n} H_{n+1}-C_{n} H_{p}+D_{n}\right\} \\
& -E_{n} H_{p+n+1}+F_{n},
\end{aligned}
$$

$$
\text { where } \begin{array}{ll}
A_{n}=\frac{n^{2}+3 n+p n+p+1}{(p+n+2)(p+n+3)}, & B_{n}=\frac{1}{(p+n+2)^{2}}-\frac{n+2}{(p+n+3)^{2}}, \\
C_{n}=\frac{p+n+1}{(p+n+2)^{2}}-\frac{p+1}{(p+n+3)^{2}}, & D_{n}=\frac{p+n}{(p+n+2)^{3}}-\frac{p-n-1}{(p+n+3)^{3}} \\
E_{n}=\frac{(p+n+1)^{2}}{(p+n+2)^{2}}-\frac{(p+n+1)(p+n+2)}{(p+n+3)^{2}}, & F_{n}=\frac{(p+n)(p+n+1)}{(p+n+2)^{3}}-\frac{(p+n+1)^{2}}{(p+n+3)^{3}} .
\end{array}
$$

When $p=0$, Corollary 22 reduces to the interesting identity:

$$
\begin{aligned}
\sum_{k=1}^{n} \frac{(-1)^{k}}{\left(\begin{array}{l}
n \\
k
\end{array}\right)} k H_{k} H_{n-k} & =\left\{\frac{3 n+5}{(n+3)^{3}}-\frac{2 n+2}{(n+2)^{3}}\right\}\left\{1+(-1)^{n} \frac{\left(n^{2}+5 n+7\right)\left(n^{3}+3 n^{2}+2 n+2\right)}{n^{4}+3 n^{3}-6 n^{2}-24 n-14}\right\} \\
& -\frac{(n+1)\left(n^{2}+3 n+1\right)}{(n+2)^{2}(n+3)^{2}}\left\{1+(-1)^{n} \frac{n^{3}+5 n^{2}+6 n-1}{n^{2}+3 n+1}\right\} H_{n} .
\end{aligned}
$$

Setting $p=n$ in Corollary 22, we attain the identity:

$$
\begin{aligned}
\sum_{k=1}^{n} \frac{(-1)^{k}}{\left(\begin{array}{c}
2 n \\
k
\end{array}\right)} k H_{k} H_{2 n-k} & =\frac{(-1)^{n}}{\left(\begin{array}{c}
2 n \\
n
\end{array}\right)}\left\{\frac{2 n^{2}+4 n+1}{4 n+6} H_{n}^{2}+\frac{H_{n}}{2(2 n+3)^{2}}+\frac{4 n^{2}+10 n+7}{4(n+1)(2 n+3)^{3}}\right\} \\
& -\frac{(2 n+1)\left(4 n^{2}+6 n+1\right)}{4\left(2 n^{2}+5 n+3\right)^{2}} H_{2 n}-\frac{2 n+1}{4(n+1)^{3}}+\frac{6 n+5}{(2 n+3)^{3}} .
\end{aligned}
$$

Making $p \rightarrow p+1$ for Corollary 22 and considering the relation:

$$
\begin{aligned}
\sum_{k=1}^{n} \frac{(-1)^{k}}{\left(\begin{array}{c}
p+n+1 \\
k
\end{array}\right)} k H_{k} H_{p+n+1-k} & =\sum_{k=1}^{n} \frac{(-1)^{k}}{\left(\begin{array}{c}
p+n \\
k
\end{array}\right)}\left(k-\frac{k^{2}}{p+n+1}\right) H_{k} H_{p+n-k} \\
& +\frac{1}{p+n+1} \sum_{k=1}^{n} \frac{(-1)^{k}}{\left(\begin{array}{c}
p+n \\
k
\end{array}\right)} k H_{k},
\end{aligned}
$$

we get the following equation by using Corollaries 10 and 22 .

Corollary 23. For $p \in N_{0}$, there holds the harmonic number identity:

$$
\begin{aligned}
& \qquad \begin{aligned}
\sum_{k=1}^{n} \frac{(-1)^{k}}{\left(\begin{array}{c}
p+n \\
k
\end{array}\right)} k^{2} H_{k} H_{p+n-k} & =(-1)^{n} \frac{n+1}{\left(\begin{array}{c}
p+n \\
n
\end{array}\right)}\left\{R_{n} H_{n+1} H_{p}-S_{n} H_{n+1}-T_{n} H_{p}-U_{n}\right\} \\
& +V_{n} H_{p+n+1}-W_{n},
\end{aligned} \\
& \text { where } \quad \begin{aligned}
R_{n}= & \frac{1}{p+n+2}-\frac{3 n+6}{p+n+3}+\frac{(n+2)(n+3)}{p+n+4}, \\
S_{n}= & \frac{1}{(p+n+2)^{2}}-\frac{3 n+6}{(p+n+3)^{2}}+\frac{(n+2)(n+3)}{(p+n+4)^{2}}, \\
T_{n}= & \frac{(p+n+1)^{2}}{(p+n+2)^{2}}-\frac{(p+1)(2 p+2 n+3)}{(p+n+3)^{2}}+\frac{(p+1)(p+2)}{(p+n+4)^{2}}, \\
U_{n}= & \frac{2 p+2 n+2}{(p+n+2)^{3}}-\frac{2 n^{2}+7 n+2 p n+7 p+9}{(p+n+3)^{3}}+\frac{3 n+2 p n+5 p+8}{(p+n+4)^{3}} \\
V_{n}= & \left\{\frac{2 p+2 n+3}{(p+n+2)^{2}}-\frac{5 p+5 n+12}{(p+n+3)^{2}}+\frac{3 p+3 n+10}{(p+n+4)^{2}}\right\}(p+n+1), \\
W_{n}= & \left\{\frac{2 p+2 n+2}{(p+n+2)^{3}}-\frac{5 p+5 n+9}{(p+n+3)^{3}}+\frac{3 p+3 n+8}{(p+n+4)^{3}}\right\}(p+n+1) .
\end{aligned}
\end{aligned}
$$


When $p=0$, Corollary 23 reduces to the interesting identity:

$$
\begin{aligned}
& \sum_{k=1}^{n} \frac{(-1)^{k}}{\left(\begin{array}{l}
n \\
k
\end{array}\right)} k^{2} H_{k} H_{n-k}=(n+1) H_{n+1} \\
& \times\left\{\left(\frac{2 n+3}{(n+2)^{2}}-\frac{5 n+12}{(n+3)^{2}}+\frac{3 n+10}{(n+4)^{2}}\right)-(-1)^{n}\left(\frac{1}{(n+2)^{2}}-\frac{3 n+6}{(n+3)^{2}}+\frac{n^{2}+5 n+6}{(n+4)^{2}}\right)\right\} \\
& -\left\{\left(\frac{2 n+2}{(n+2)^{3}}-\frac{5 n+9}{(n+3)^{3}}+\frac{3 n+8}{(n+4)^{3}}\right)+(-1)^{n}\left(\frac{2 n+2}{(n+2)^{3}}-\frac{2 n^{2}+7 n+9}{(n+3)^{3}}+\frac{3 n+8}{(n+4)^{3}}\right)\right\}(n+1) .
\end{aligned}
$$

Taking $p=n$ in Corollary 23, we gain the identity:

$$
\begin{aligned}
& \sum_{k=1}^{n} \frac{(-1)^{k}}{\left(\begin{array}{c}
2 n \\
k
\end{array}\right)} k^{2} H_{k} H_{2 n-k}=\frac{(-1)^{n} n}{\left(\begin{array}{c}
2 n \\
n
\end{array}\right)}\left\{\frac{2 n^{2}+5 n+2}{4 n+6} H_{n}^{2}+\frac{H_{n}}{(2 n+3)^{2}}+\frac{4 n^{2}+10 n+7}{(2 n+2)(2 n+3)^{3}}\right\} \\
& -\frac{(2 n+1)\left(4 n^{4}+10 n^{3}-n^{2}-14 n-6\right)}{(n+1)^{2}(2 n+3)^{2}(2 n+4)^{2}} H_{2 n}+\frac{n\left(8 n^{6}+12 n^{5}-132 n^{4}-512 n^{3}-731 n^{2}-459 n-103\right)}{4(n+1)^{3}(n+2)^{3}(2 n+3)^{3}} .
\end{aligned}
$$

\section{REFERENCES}

[1] G. E. Andrews, K. Uchimura, Identities in combinatorics IV: differentiation and harmonic numbers, Utilitas Math. 28 (1985) 265-269.

[2] Y. Chen, Q. Hou, H. Jin, The Abel-Zeilberge algorithm, Electron. J. Comb. 18 (2011) \#P17.

[3] W. Chu, Summation formulae involving harmonic numbers, Filomat 26 (2012) 143-152.

[4] F. Chyzak, An extension of Zeilberger's fast algorithm to general holonomic functions, Discrete Math. 217 (2000) 115-134.

[5] R.L. Graham, D.E. Knuth, O. Patashnik, Concrete Mathematics: a Foundation for Computer Science, Addison-Wesley Publishing Company, Amsterdam, 2nd edition, 1994.

[6] H. Liu, W. Wang, Harmonic number identities via hypergeometric series and Bell polynomials, Integral Transforms Spec. Funct. 23 (2012) 49-68.

[7] P. Paule, C. Schneider, Computer proofs of a new family of harmonic number identities, Adv. Appl. Math. 31 (2003) 359-378.

[8] C. Schneider, Symbolic summation assists Combinatorics, Sém. Lothar. Combin. 56 (2006) Article B56b.

[9] A. Sofo, Some more identities involving rational sums, Appl. Anal. Discr. Math. 2 (2008) 56-66.

[10] D. Zheng, Further summation formulae related to generalized harmonic numbers, J. Math. Anal. Appl. 335 (2007) 692-706. 